\title{
Dynamic bias and its implications for GPCR drug discovery
}

\section{Martin C. Michel, Roland Seifert and Richard A. Bond}

In their Opinion article (Signalling bias in new drug discovery: detection, quantification and therapeutic impact. Nature Rev. Drug Discov. 12, 205-216 (2013)) ${ }^{1}$, Kenakin and Christopoulos provide a detailed explanation of ligand bias, where a ligand preferentially stimulates certain $G$ proteincoupled receptor (GPCR)-activated signalling pathways over others. Although the Review acknowledges system bias and observational bias as aspects that may prevent the accurate definition of ligand bias, one aspect that was not discussed was that produced by the dynamic nature of in vivo systems.

\section{Dynamic bias: definition and examples} GPCR expression, compartmentalization and function are dynamically regulated by physiological and pathophysiological factors, as well as by treatments, and this regulation also applies to the expression of specific $G$ proteins and arrestins. Changes in such regulation will affect the stoichiometry of GPCRs with downstream signalling pathways, as well as the spontaneous level of receptor activity; together, these effects represent something we propose calling 'dynamic bias'.

Changes to a system over time that result from ageing, disease or long-term drug treatment may result in dynamic bias which may continuously change the system bias. We propose that such changes - induced by chronic treatment and/or the underlying pathology - affect the system in a manner that changes system bias and therefore ligand bias, and the subsequent receptor signalling is altered in such a way that only subsets of a therapeutic class of drugs are useful in the treatment of a disease.

Dynamic bias describes alterations of the defined system bias by factors that are outside the assay being used to measure effects on GPCR signalling. Defining ligand bias as a molecular constant requires an assessment of the system bias of the conditions at the same time at which the ligand bias is measured, and is therefore a static 'snapshot' of the system at a given time. Dynamic bias, however, encompasses factors that will continuously affect system bias and that therefore severely limit the possibility of obtaining a molecular constant for ligand bias. Dynamic bias may have limited effects in very reductionist cell-based assays that are relatively static, but assays of greater complexity would most definitely not represent a static system because of the effects of dynamic bias. The concept of dynamic bias could help further understanding of known phenomena such as why there are subsets of drugs within a therapeutic class that have disease-specific beneficial effects that are not fully explained by correlation to results obtained from in vitro assays.

\section{Dynamic bias owing to pathological}

changes. In congestive heart failure ( $\mathrm{CHF})$, the disease itself is characterized by: a desensitization and downregulation of $\beta_{1}$-adrenoceptors and the desensitization of $\beta_{2}$-adrenoceptors; a downregulation of $\mathrm{G}_{\mathrm{s}}$ protein; and an upregulation of $\mathrm{G}_{\mathrm{i}}$ protein, $\beta$-arrestin 1 and GPCR kinases ${ }^{2}$. Together, these changes not only shift the balance of $\beta_{1}$ - and $\beta_{2}$-adrenoceptor stimulation but also dampen the effects of the $\beta_{1}$-adrenoceptor$\mathrm{G}_{\mathrm{s}}$-cyclic-AMP pathway and promote $\mathrm{G}_{\mathrm{i}}$ - and arrestin-dependent signalling.

Such pathology-driven dynamic bias offers a potential explanation for why some $\beta$-adrenoceptor antagonists had beneficial effects on survival of individuals with CHF, whereas use of other $\beta$-adrenoceptor antagonists lacked a beneficial outcome or even had detrimental effects. In cardiomyocytes from CHF patients, inverse $\beta$-adrenoceptor agonists (including carvedilol and metoprolol) have beneficial effects, whereas in vivo, neutral antagonists or weak partial agonists (such as bucindolol and celiprolol) lack beneficial effects ${ }^{3,4}$. These results suggest that inverse agonism is essential for therapeutic success in the treatment of $\mathrm{CHF}$.

This is a surprising conclusion, because constitutive activity is required for inverse agonism to become detectable and relevant, and yet heart failure is a condition in which any such constitutive activity would be expected to be largely eliminated ${ }^{5}$. However, in $\mathrm{CHO}$ (Chinese hamster ovary) cells that were transfected with $\beta_{2}$-adrenoceptors, pre-incubation with the non-selective $\beta$-adrenergic agonist isoprenaline made inverse agonist properties easier to detect ${ }^{6}$. So, the high sympathetic drive in CHF may enhance differences between neutral antagonists and inverse agonists, creating a dynamic bias.

Moreover, in a post-approval headto-head trial of carvedilol and metoprolol for the treatment of CHF, carvedilol demonstrated a greater reduction in mortality (reported in the COMET study) ${ }^{7}$, prompting speculation as to what differentiates carvedilol from metoprolol. Biased signalling was one proposed differentiating factor ${ }^{8}$. Several studies have shown that although both carvedilol and metoprolol are inverse agonists at the canonical $\beta$-adrenoceptor- $G_{s}$ pathway, carvedilol can stimulate extracellular signal-regulated kinase 1 (ERK1) or ERK2 signalling via a $\beta$-arrestin-dependent pathway ${ }^{4,8,9}$ that has been shown to be cardio-protective in animal models of heart failure ${ }^{8}$.

Dynamic bias owing to chronic treatment. Agonists and antagonists can acutely stimulate and inhibit GPCR function, but chronically cause downregulation and upregulation of the same GPCR function, respectively. Accordingly, GPCR ligands can have opposite effects upon acute and chronic administration. For instance, luteinizing hormone-releasing hormone receptor agonists that are commonly used to treat hormone-sensitive prostate cancer acutely elevate testosterone levels, but their chronic use lowers plasma testosterone to near-castration levels ${ }^{10}$. Similarly, the sphingosine-1-phosphate receptor agonist fingolimod (FTY-720) acutely activates lymphocytes but chronically depresses immune function and thus represents a valuable treatment for multiple sclerosis ${ }^{11}$.

Acutely administered $\beta_{2}$-adrenoceptor agonists are key treatments for asthma; however, chronic use of long-acting $\beta_{2}$-adrenoceptor agonists has been associated with a loss of asthma-symptom control and a small but significant increase in asthma-related mortality ${ }^{12,13}$. Conversely, $\beta_{2}$-adrenoceptor antagonists acutely worsen lung function in patients with asthma, but upon chronic use, some of these antagonists may improve lung function ${ }^{14}$. $\beta$-adrenoceptor antagonists acutely impair cardiac function ${ }^{15}$ but, when administered chronically to patients with $\mathrm{CHF}$, some members of this drug class can improve cardiac contractility ${ }^{15}$ and have dramatically beneficial effects on survival ${ }^{16,17}$. 


\section{Implications for drug discovery}

Dynamic bias as an explanation of differential drug effects in ageing, disease or with chronic treatment is speculative but plausible, and it has obvious implications for drug development. The reversal of responses in the cases above demonstrate the influence of a factor that is more or less ignored in high-throughput drug discovery assays: time. Furthermore, because the reversal of response is often a compensatory effect of the whole organism, and is probably not detectable in cell-based assays, such effects cannot be mimicked simply by prolonging the treatment time in high-throughput assays. Indeed, in many cases the drug has to be at equilibrium with its molecular target for several days or weeks before a therapeutic effect is observed.

Therefore, besides awareness of system bias, future assays for ligand bias in drug discovery will have to account for dynamic bias. In order to optimize future drug development and to take into consideration the complexity of signal-transduction pathways that are activated by any given ligand, the pharmaceutical industry now implements assay platforms in which multiple parameters are simultaneously monitored. The early use of in vivo models that are hopefully representative of human disease situations is another approach that is now being used to reveal and investigate such complexities. Although these strategies may lead to a better selection of drug development candidates that are disease-specific yet share the same primary molecular target, defining the parameters for such selection poses many challenges.

Martin C. Michel is at the Department of Pharmacology, Johannes Gutenberg University, Obere Zahlbacher Strasse 67, 51101 Mainz, Germany.

Roland Seifert is at the Department of Pharmacology, Hannover Medical School, Carl-Neuberg-Strasse 1, D-30625 Hannover, Germany.

Richard A. Bond is at the Department of Pharmacology and Pharmaceutical Sciences, Science and Research Building 2, Room 521, University of Houston, Houston, Texas 77204-5037, USA. Correspondence to R.A.B. e-mail: RABond@uh.edu doi: 10.1038/nrd3954-c3
1. Kenakin, T. \& Christopoulos, A. Signalling bias in new drug discovery: detection, quantification and therapeutic impact. Nature Rev. Drug Discov. 12, 205-216 (2013).

2. Brodde, O. -E. $\beta$-Adrenoceptor blocker treatment and the cardiac $\beta$-adrenoceptor-G-protein(s)-adenylyl cyclase system in chronic heart failure. Naunyn Schmiedebergs Arch. Pharmacol. 374, 361-372 (2007).

3. Maack, C. et al. Characterization of $\beta$-selectivity, adrenoceptor- $\mathrm{G}_{\mathrm{s}}$-protein interaction and inverse agonism of nebivolol in human myocardium. Br. J. Pharmacol. 132, 1817-1826 (2001).

4. Galandrin, S. \& Bouvier, M. Distinct signaling profiles of $\beta_{1}$ and $\beta_{2}$ adrenergic receptor ligands towards adenylyl cyclase and mitogen-activated protein kinase reveals pluridimensionality of efficacy. Mol. Pharmacol. 70, 1575-1584 (2006).

5. Brodde, O. -E. \& Michel, M. C. Adrenergic and muscarinic receptors in the human heart. Pharmacol. Rev. 51, 651-689 (1999).

6. Chidiac, P., Hebert, T. E., Valiquette, M., Dennis, M $\&$ Bouvier, M. Inverse agonist activity of $\beta$-adrenergic antagonists. Mol. Pharmacol. 45, 490-499 (1994).

7. Poole-Wilson, P. A. et al. Comparison of carvedilol and metoprolol outcomes in patients with chronic heart failure in the carvedilol or metoprolol European trial (COMET): randomised controlled trial. Lancet 362 , 7-13 (2003).

8. Wisler, J. W. et al. A unique mechanism of $\beta$-blocker action: carvedilol stimulates $\beta$-arrestin. Proc. Natl Acad. Sci. USA 104, 16657-16662 (2007).

9. Stallaert, W., Dorn, J. F., van der Westhuizen, E., Audet, M. \& Bouvier, M. Impedance responses reveal $\beta_{2}$-adrenergic receptor signaling pluridimensionality and allow classification of ligands with distinct signaling profiles. PLOS ONE 7, e29420 (2012).

10. Mottet, N. et al. EAU guidelines on prostate cancer. Part II: treatment of advanced, relapsing, and castration-resistant prostate cancer. Eur. Urol. $\mathbf{5 9}$ 572-583 (2011).

11. Brinkmann, V. et al. Fingolimod (FTY720): discovery and development of an oral drug to treat multiple sclerosis. Nature Rev. Drug Discov. 9, 883-897 (2010).

12. Nelson, H. S., Weiss, S. T., Bleecker, E. R., Yancey, S. W. \& Dorinsky, P. M. The salmeterol multicenter asthma research trial: a comparison of usual pharmacotherapy for asthma or usual pharmacotherapy plus salmeterol. Chest 129 , 15-26 (2006).

13. Walker, J. K., Penn, R. B., Hanania, N. A., Dickey, B. F. $\&$ Bond, R. A. New perspectives regarding $\beta_{2}$-adrenoceptor ligands in the treatment of asthma. Br. J. Pharmacol. 163, 18-28 (2011).

14. Nguyen, L. P. et al. $\beta_{2}$-adrenoceptor signaling is required for the development of an asthma phenotype in a murine model. Proc. Natl Acad. Sci. USA 106, 2435-2440 (2009).

15. Hall, S. A. et al. Time course of improvement in left ventricular function, mass and geometry in patients with congestive heart failure treated with $\beta$-adrenergic blockade. J. Am. Coll. Cardiol. 25, 1154-1161 (1995).

16. Waagstein, F. et al. Beneficial effects of metoprolol in idiopathic dilated cardiomyopathy. Lancet 342 , 1441-1446 (1993).

17. Packer, M. et al. The effect of carvedilol on morbidity and mortality in patients with chronic heart failure. N. Engl. J. Med. 334, 1349-1355 (1996).

Competing interests statement

The authors declare competing interests: see Web version for details. 